\title{
Einfach arbeiten um zu helfen
}

\section{Andreas Schneider}

Dr. med., Facharzt für Allgemeine Innere Medizin, Frauenfeld

Nach mehr als einem Jahr Vorbereitung, unter anderem mit einem zweiwöchigen Einführungskurs in Wien, reise ich Anfang März 2015 für einen humanitären Einsatz mit «Médecins Sans Frontières / Ärzte ohne Grenzen (MSF)» in den Osten der Demokratischen Republik Kongo. Ein Traum geht für mich in Erfüllung. Während

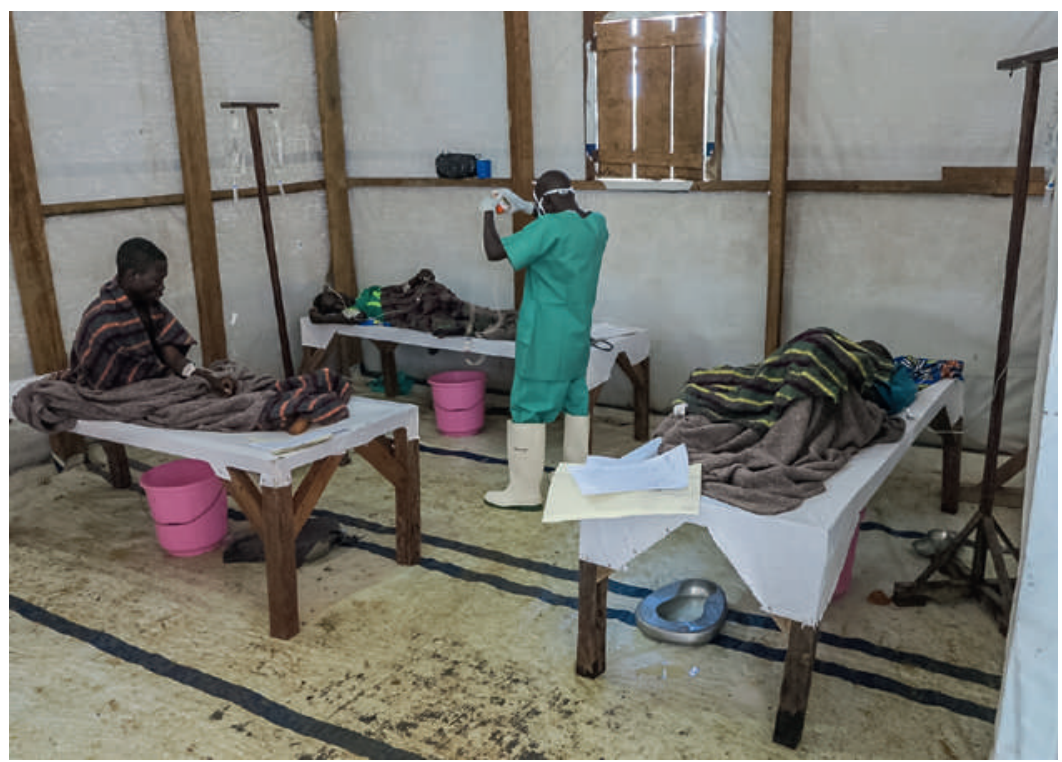

Eindruckvolles Erlebnis: der Ausbruch einer Cholera-Epidemie. Die Patienten werden isoliert, alles klappt einwandfrei.

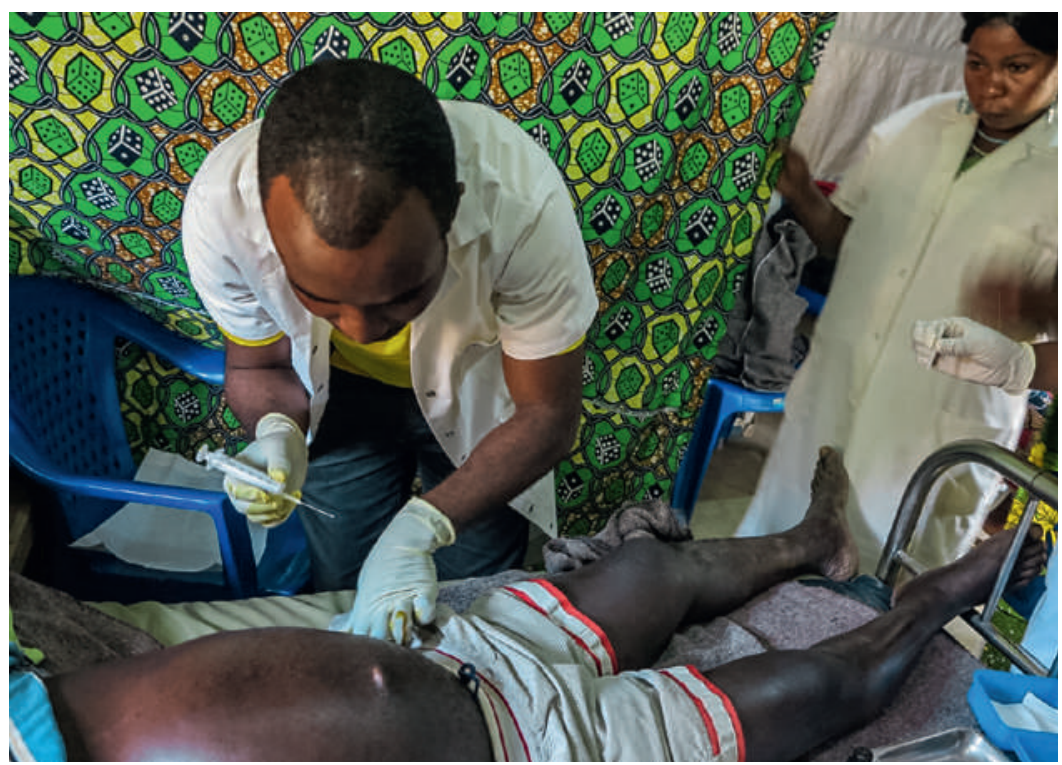

Bewundernswert: Einsatz und Fähigkeiten der kongolesischen Ärzte und des Pflegepersonals.
Jahren habe ich MSF beobachtet und immer wieder bewundert für ihre mutigen Einsätze zugunsten benachteiligter Menschen, ihre kompromisslose, humanistische Grundhaltung.

Bereits die Anreise nach Kampala, der Hauptstadt Ugandas, mit einem Kleinflugzeug über den ostafrikanischen Grabenbruch nach Bunia und danach mit einem $4 \times 4$-Fahrzeug nach Geti, ist ein unvergessliches Erlebnis. Geti ist eine afrikanische Ortschaft mit einem kleinen Spital, zwei Kirchen, einer Schule, einem Dorfzentrum, einem Marktplatz und einem Fussballfeld. Insgesamt leben etwa 200000 Menschen in der Gegend, davon

\section{Es gibt keine Sonn-, keine Feiertage.}

Es wird einfach gearbeitet.

sind die Hälfte Flüchtlinge, die seit dem Genozid in Ruanda 1994 in Lagern hausen. Diese etwa 90000 Menschen haben fast nichts, vor allem haben sie keine Zukunftsperspektiven. Ausserdem kommt es immer wieder zu bewaffneten Auseinandersetzungen. Raub, Messerstechereien, Schiessereien und Vergewaltigungen gehören zum täglichen Leben. MSF betreut die Vertriebenen und die ansässige Bevölkerung medizinisch und kümmert sich besonders um Frauen und Kinder unter fünf Jahren.

Gleich nach der Ankunft werden mir die Sicherheitsmassnahmen erklärt. Man darf sich nicht frei bewegen, die Gegend ist unsicher. Meinen geliebten Laufsport werde ich nicht ausüben können. Durch einen Rundgang lerne ich das umzäunte Camp kennen und kann mein kleines Doppelzimmer beziehen. Die einfache Unterkunft ist reduziert auf das Wesentliche, kein Luxus. Danach Fahrt zum Spital und erste Besichtigung meines neuen Arbeitsplatzes.

Im «Salle d'urgence et soins intensifs», kurz: «SUSI» soll ich in den nächsten Wochen einen kongolesischen Arztkollegen unterstützen. Das Gebäude, die Einrichtungen, die vorhandenen diagnostischen und therapeutischen Mittel sind sehr einfach, aber sauber und zweckmässig. Sofort werde ich vom Team mitgenommen, bin bei der Untersuchung von Kindern mit schwerer Malaria dabei, erlerne die entsprechenden therapeutischen Massnahmen. Beurteile Patienten verschiedenen Alters mit Meningitiden, mache Lumbalpunktionen und verordne die notwendigen Antibiotika. Mangelernährte Kinder, schwerste septische Infektionen, Tuberkulose, AIDS, 


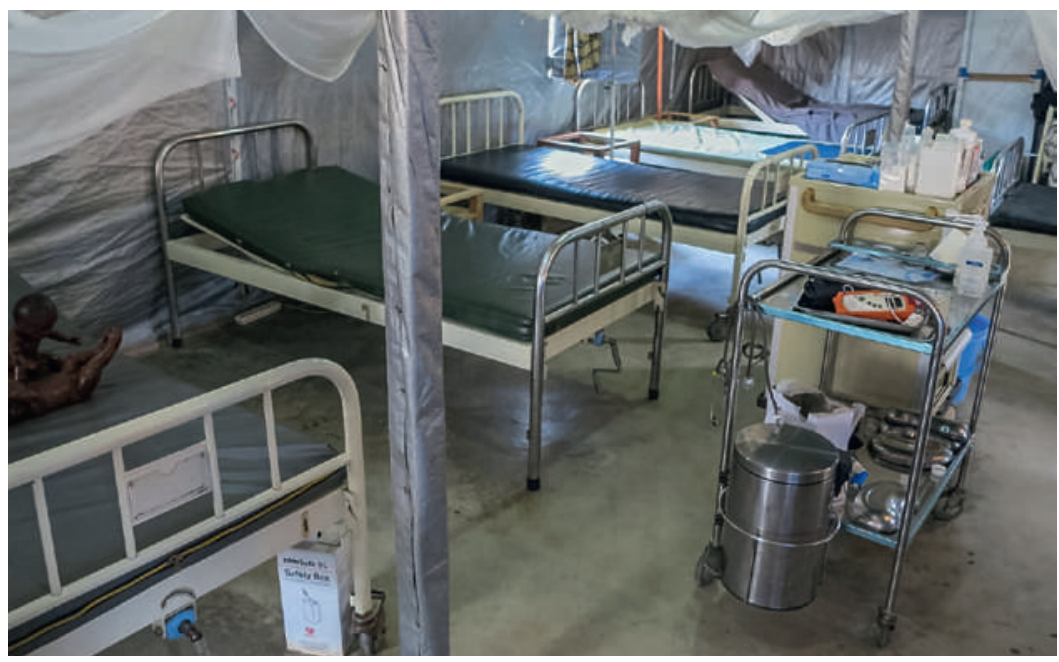

Gebäude, Einrichtung, diagnostische und therapeutische Mittel sind einfach, aber sauber und zweckmässig.

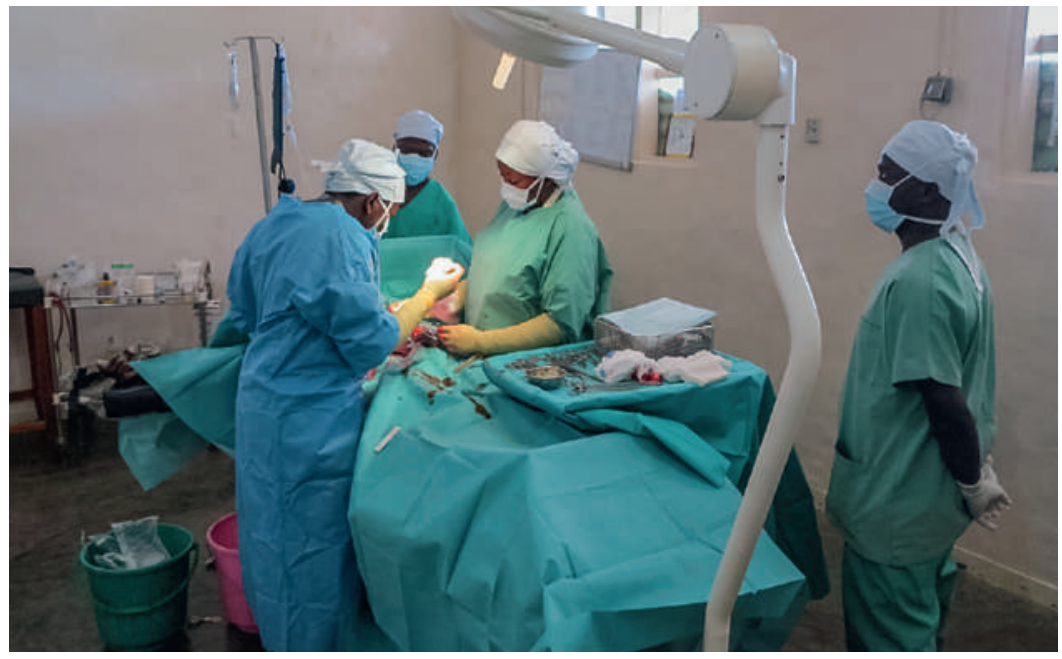

Auch bei grösster Arbeitsbelastung wird rasch und mit hoher medizinischer Professionalität gehandelt.

Korrespondenz: Dr. med. Andreas Schneider Bahnhofstrasse 61 CH-8501 Frauenfeld Tel. 0527284043
Vergiftungen, Verletzungen als Folge gewalttätiger Auseinandersetzungen, aber auch ein entgleister Diabetes mellitus, Schlaganfälle, eingeklemmte Leistenbrüche gehören zu unserer Arbeit.

Jeder Tag läuft gleich ab, es gibt keine Sonn-, keine Feiertage. Es wird einfach gearbeitet, man versucht den Menschen zu helfen. Immer wieder auch sehr traurige Situationen, eine Mutter bringt ihr Kleinkind auf dem Arm ins Spital, sie ist etwa 15 Kilometer gelaufen, wir können nur den Tod feststellen. Der Tod gehört hier zum Leben, wie die Nacht zum Tag, er wird als Selbstverständlichkeit akzeptiert.

Der Einsatz und die Fähigkeiten der kongolesischen Arztkollegen und des Pflegepersonals sind bewundernswert. Auch bei grössten Arbeitsbelastungen gibt es keine

Bei der Behandlung von Bagatellerkrankungen denke ich immer wieder an die leidenden Menschen, vor allem an die Kinder.

Klagen, keine Hektik, es wird rasch und zielgerichtet gehandelt mit hoher medizinischer Professionalität. Mit grosser Geduld werden mir die verschiedenen tropischen Krankheitsbilder und ihre möglichen Therapien erklärt. Selten habe ich so viel Neues gelernt wie in diesen Wochen.

Besonders prägend war für mich auch der Ausbruch einer Choleraepidemie. Innerhalb des ersten Tages erkranken etwa 30 Personen. Sofort wird eine entsprechende Isolationsstation eingerichtet und die Patienten werden dort versorgt. Für jeden Erkrankten benötigen wir täglich 10 Liter sterile Infusionen, das heisst etwa 300 Liter Ringerlactat im Tag. Die Logistik klappte einwandfrei.

Leider entwickelte sich in meinem rechten Bein eine ausgedehnte Thrombose vom Fuss bis in die Leiste, Folge einer Zerrung, des langen Stehens im Spital, der fehlenden Bewegung, der Wärme, der knappen Trinkmenge. Eine Behandlung in Geti ist nicht möglich, ein Verbleiben wegen der Gefahr einer Lungenembolie, respektive eines bleibenden Schadens am Bein ebenfalls nicht. Eine medizinische Evakuation in ein Spital von Nairobi ist die Folge und danach ein ärztlich begleiteter Rückflug in die Schweiz.

Die Enttäuschung über den vorzeitigen Abbruch der Tätigkeit in Geti ist gross. Zum Glück erhole ich mich sehr rasch. In der Zwischenzeit kann ich wieder meiner gewohnten Arbeit als Hausarzt in einer Grosspraxis in Frauenfeld nachgehen. Bei der Behandlung von Bagatellerkrankungen denke ich immer wieder an die leidenden Menschen, vor allem an die schwerkranken Kinder in Geti.

Bildnachweis

Alle Bilder @ Andreas Schneider 